\title{
Temporal changes in nasopharyngeal carriage of Streptococcus pneumoniae serotype 1 genotypes in healthy Gambians before and after the 7-valent pneumococcal conjugate vaccine
}

Chinelo Ebruke, Anna Roca, Uzochukwu Egere, Ousainou Darboe, Philip C Hill, Brian Greenwood, Brendan W Wren, Richard A Adegbola, Martin Antonio

Streptococcus pneumoniae serotype 1 is one of the leading causes of invasive pneumococcal disease. However this invasive serotype is hardly found in nasopharyngeal asymptomatic carriage and therefore large epidemiological studies are needed to assess the dynamics of serotype 1 infection. Within the context of a large cluster randomized trial conducted in rural Gambia to assess the impact of PCV-7 vaccination on nasopharyngeal carriage, we present an ancillary analysis describing the prevalence of nasopharyngeal carriage of pneumococcal serotype 1 and temporal changes of its more frequent genotypes.

Nasopharyngeal swabs (NPS) were collected before PCV-7 vaccination (December 2003May 2004) and up to 30 months after PCV-7 vaccination. The post-vaccination time was divided in three periods to ensure an equal distribution of the number of samples: (1) July 2006 - March 2007, (2) April 2007 - March 2008 and (3) April 2008 - Feb 2009). S. pneumoniae serotype 1 were genotyped by MLST.

Serotype 1 was recovered from $87(0.71 \%)$ of 12,319 NPS samples collected. In the prevaccination period, prevalence of serotype 1 was $0.47 \%$ in both study arms. In the postvaccination periods, prevalence in the fully vaccinated villages ranged between $0.08 \%$ in period 1 and $0.165 \%$ in period 2 ; while prevalence in partly vaccinated villages was between $0.17 \%$ in period 3 and $1.34 \%$ in period 2 . Overall four different genotypes were obtained with ST3081 the most prevalent $(60.71 \%)$ followed by ST618 $(29.76 \%)$. ST3081 was found only in post-vaccination period 2 and 3 while ST618 had disappeared in postvaccination period 3. Distribution of these major genotypes was similar in both study arms.

Emergence of ST3081 and concomitant disappearance of ST618 may suggest a change in the molecular epidemiology of pneumococcal serotype 1 in this region. This change is not 
likely to be associated with the introduction of PCV-7 which lacks serotype 1 as it was observed simultaneously in both study arms. Future population-based epidemiological studies will provide further evidence of substantive changes in the pneumococcal serotype 1 epidemiology and the likely mechanisms. 
2 Chinelo Ebruke ${ }^{1,2}$

3 Anna Roca ${ }^{1}$

4 Uzochukwu Egere ${ }^{1}$

5 Ousainou Darboe ${ }^{1}$

6 Philip C Hill ${ }^{3}$

7 Brian Greenwood ${ }^{2}$

8 Brendan W. Wren ${ }^{2}$

9 Richard A Adegbola ${ }^{1,4}$

10 Martin Antonio ${ }^{1,2,5 *}$

11

$12{ }^{1}$ Vaccinology Theme, Medical Research Council Unit, Banjul, The Gambia

13 2Faculty of Infectious and Tropical Diseases, London School of Hygiene \& Tropical Medicine,

14 London, United Kingdom

$15{ }^{3}$ Centre for International Health, School of Medicine, University of Otago, New Zealand

$16 \quad{ }^{4}$ GlaxoSmithKline Biologicals Wavre, Belgium

$17{ }^{5}$ Microbiology and Infection Unit, Warwick Medical School, University of

18 Warwick, Coventry, United Kingdom

19 *For corresponding author email: mantonio@mrc.gm 


\section{Introduction}

21 Streptococcus pneumoniae is usually found in the nasopharynx of healthy individuals which is

22 considered a necessary step preceding invasive pneumococcal disease (IPD), including pneumonia,

23 meningitis, and bacteraemia (Gleich, Morad et al. 2000, Bogaert, De Groot et al. 2004, Baker, Barrozo

24 et al. 2005, Adegbola, Hill et al. 2006, Hill, Akisanya et al. 2006, Ahern and Raszka 2009, Balicer,

25 Zarka et al. 2010). There are over 90 different $S$. pneumoniae serotypes of which serotype 1 is a

26 common cause of IPD worldwide with particular high rates of disease in sub-Saharan Africa

27 (Hausdorff, Bryant et al. 2000, Adegbola, Hill et al. 2006, Gessner, Mueller et al. 2010).

28 As cases of invasive disease represent only a small fraction of the pneumococcal burden, there is an

29 increasing interest on evaluating the prevalence of pneumococcal asymptomatic carriage in the

30 nasopharynx, since this is important in understanding the dynamics of disease and transmission as well

31 as providing a basis for assessing the impact of interventions (Bogaert, De Groot et al. 2004).

32 Interestingly, serotype 1 is rarely found in the nasopharynx of healthy individuals with prevalence

33 always below 1-2\% (Hill, Akisanya et al. 2006, Laval, de Andrade et al. 2006, Antonio, Hakeem et al.

34 2008, Nunes, Sa-Leao et al. 2008). As a result, there are just few studies evaluating the dynamics of

35 serotype 1 in nasopharyngeal carriage as large epidemiological studies are needed.

37 Although PCV-7, the first licensed pneumococcal vaccine, did not include serotype 1 in its formulation

38 new PCV licensed vaccines (PCV-10 and PCV-13) include this serotype and therefore, understanding

39 the dynamics of serotype 1 carriage has become a priority. Within the context of a cluster-randomized

40 trial conducted in rural Gambia (Roca, Hill et al. 2011), we collected a large number of NPS (12, 319

41 samples) before and up to 30 months after starting the trial (Hill, Akisanya et al. 2006, Roca, Hill et al. 
42 2011). As an ancillary study of the trial, we describe the dynamics of pneumococcal serotype 1

43 nasopharyngeal carriage within a period of 6 years before and after the PCV-7 trial.

44

45 


\section{Materials and Methods}

\section{Study design and collection of isolates}

48 This study was an ancillary study of large pneumococcal carriage studies conducted in 21 selected

49 villages in rural Gambia as previously described (Hill, Akisanya et al. 2006, Roca, Hill et al. 2011).

50 Firstly, a pre-vaccination cross sectional survey was conducted between December 2003 and May 2004

51 in which NP samples were collected from subjects of all age groups (Hill, Akisanya et al. 2006).

52 Following this, a single-blind, cluster-randomized (by village) trial to evaluate the impact of PCV-7 on

53 pneumococcal carriage was conducted in the study villages (Roca, Hill et al. 2011). In one group of 11

54 villages, all individuals over the age of 30 months received one dose of PCV-7 whilst subjects in this

55 age group resident in 10 control villages received one dose of serogroup $\mathrm{C}$ meningococcal conjugate

56 vaccine. All children less than 30 months of age in both study groups and infants born during the

57 course of the trial received PCV-7. The trial showed a marked decrease of nasopharyngeal carriage of

58 vaccine type (VT) pneumococci in all age groups and both study arms with a more marked drop in

59 villages where the whole community had received PCV-7 (Roca, Hill et al. 2011). There was little

60 change in the overall prevalence of NVT carriage following introduction of the vaccine (Roca, Hill et

61 al. 2011).

62

63 NP swabs were collected as part of several ongoing studies. First, a large pre-vaccination cross-

64 sectional survey (CSS) conducted in 2003-2004, prior to a PCV-7 randomized trial (Hill, Akisanya et

65 al. 2006). Later, as part of the cluster-randomized PCV-7 trial, NPS were collected in three different

66 cross-sectional surveys (from 4-6 months, 12-14 months and up to 30 months) (Roca, Hill et al. 2011)

67 and as well as a longitudinal study between 4 and 30 months (unpublished data) after vaccination

68 (Table 1). For the purposes of this analysis, post-vaccination data were shown stratified in three 
69 different time-periods defined to ensure at least 2,000 samples in each period as the number of samples 70 were not equally distributed throughout the follow up period. (Table1).

72 Approval for this study was obtained from the Joint Medical Research Council (MRC)/Gambia

73 Government Ethics Committee and the Ethics Committee of the London School of Hygiene \& Tropical

74 Medicine, UK (SCC number 1032, ISRCTN 51695599). Community and individual consent was

75 obtained from study participants and the conduct of the trial was guided by a Data Safety and

76 Monitoring Board.

77

78 Serotyping and multi locus sequence typing (MLST)

79 A total of 87 S. pneumoniae serotype 1 isolates obtained from NP swabs during a survey were

80 identified by latex agglutination (Hill, Akisanya et al. 2006) and confirmed by molecular serotyping

81 (Morais, Carvalho Mda et al. 2007). Multi locus sequence typing was performed on viable $S$.

82 pneumoniae serotype 1 isolates recovered after storage at $-70^{\circ} \mathrm{C}$ as previously described (Antonio,

83 Hakeem et al. 2008).

84

\section{Data analysis}

86 All statistical analysis were carried out in STATA (version 11, Stata m Corporation, College Station

87 TX) using Chi-square tests. $p$-Values less than 0.05 were taken to indicate statistical significance. Wet 88 season was considered from June to October each year. Sequences were edited and aligned using the 89 Laser Gene DNA star 7.1 software. Sequence type (ST) was obtained by submission of sequences onto 90 the MLST database website. 


\section{Results}

92 A total of 12,319 NP samples were collected during the study; $22.2 \%$ of which were from the pre-

93 vaccination period and $32.4 \%, 28.2 \%$ and $17.2 \%$ from the post-vaccination study periods 1 to 3

94 respectively. The median age of sampled individuals was 15 years (IQR 5.9 - 45 years), 11 years (IQR

954.7 - 29 years), 11 years (IQR 5.6 - 27 years) and 14 years (IQR 6.7 - 33 years), in the pre-vaccination

96 and post vaccination periods 1 to 3 respectively. The overall prevalence of $S$. pneumoniae in the pre-

97 vaccination period was $71.78 \%$ (1,971 out of 2,746 samples). The overall prevalence of S. pneumoniae

98 in the post-vaccination periods 1 to 3 was $47.08 \%(4,507$ out of 9573 samples).

99

100 The overall prevalence of $S$. pneumoniae serotype 1 was $0.71 \%$ ( 87 of 12,319 samples collected).

101 Prevalence of serotype 1 carriage was highest (1.02\%) among children aged 5 to 14 years $(\mathrm{p}<0.001)$

102 compared to other age groups. Serotype 1 carriage prevalence was highest in post-vaccination period 2

$103(1.50 \%)$ compared to the other study periods $(\mathrm{p}<0.001)$ (Table 1$)$.

104 Serotype 1 isolates were likely to be found during the wet season $0.81 \%$ (40 out of 4,909 samples

105 collected) compared to the dry season $0.64 \%$ (47 out of 7,323 samples collected) $(p=0.268)$ but these

106 were not statistically significant.

107

108 The overall prevalence of serotype 1 pneumococcal carriage was similar in vaccinated and control

109 villages $(0.73 \%$ versus $0.68 \% ; p=0.703)$. In the pre-vaccination study period, prevalence of carriage of

110 serotype 1 was the same in both vaccinated and control villages $(0.47 \%$ each $)$. However, the

111 prevalence was lower in vaccinated than in control villages in post-vaccination period $1(0.08 \%$ vs.

$1120.48 \%, p=0.011)$, similar in vaccinated and control villages in period $2(1.65 \%$ vs. $1.34 \%, p=0.459)$

113 and higher in vaccinated villages in period $3(1.16 \%$ vs. $0.17 \%, p=0.004)$ (Table 2$)$. 
115 MLST analysis was performed for 84 of the 87 serotype 1 isolates obtained (97\%). Four different STs

116 were obtained with ST3081 being the predominant ST $(60.71 \%)$ in both vaccinated and control villages

117 followed by ST618 (29.76\%), ST217 (7.14\%) and ST303 (2.38\%). Prevalence of different STs was not

118 associated with age groups $(p=0.368)$. However, the distribution of STs differed over the course of the

119 study $(\mathrm{p}<0.001)$. ST3081 was seen only in the post vaccination periods. ST618 was seen in the pre-

120 vaccination and periods 1 and 2 post vaccination, but not in the post vaccination period 3 (Figure 1).

121 Differences in the distribution of ST over the study periods was apparent in both vaccinated $(p=0.002)$

122 and control $(p=0.021)$ villages (Figure 2$)$, with the observed expansion of ST3081 and the

123 disappearance of ST618 occurring in both groups (Figures 1 \& 2).

124

125 


\section{Discussion}

127 Given that pneumococcal serotype 1 is one of the common cause of IPD worldwide and the paradox of

128 its' rarity in nasopharyngeal carriage, it is not unsurprising that only a few published studies have

129 evaluated serotype 1 carriage patterns. To our knowledge, this is the largest study evaluating the

130 dynamics of pneumococcal serotype 1 carriage. We present findings from as many as 87 serotype 1

131 isolates and report on the prevalence and dominant genotype patterns over a 6 year period. The finding

132 of $0.71 \%$ overall prevalence in carriage of serotype 1 agrees with earlier findings indicating the rarity

133 of serotype 1 in carriage studies (Brueggemann and Spratt 2003, Hausdorff, Feikin et al. 2005, Laval,

134 de Andrade et al. 2006, Nunes, Sa-Leao et al. 2008, Smith-Vaughan, Marsh et al. 2009) We note also

135 that this low carriage rate was observed in both the pre- and post vaccination periods with no

136 significant differences between study arms. However, prevalence of serotype 1 carriage was highest in

137 the age group 5-14 years. Findings from other studies suggest that this age group is at particular risk for

138 serotype 1 IPD as opposed to other serotypes (Adegbola, Hill et al. 2006, Gessner, Mueller et al. 2010).

139 Introduction of the pneumococcal conjugate vaccine PCV-7 is associated with a reduction in carriage

140 of VT serotypes but has also been linked to an increase in carriage of NVT in some settings (Mbelle,

141 Huebner et al. 1999, Huang, Platt et al. 2005, O'Brien, Millar et al. 2007) but not in our setting (Roca,

142 Hill et al. 2011) and elsewhere (Millar, Watt et al. 2008, Roca, Hill et al. 2011). In this study, serotype

1431 prevalence showed variation over the study period but this is not likely to have been related to

144 vaccine introduction as there was no consistent trend and no consistent difference between vaccinated

145 and control villages. A higher carriage rate in the vaccinated group compared to the controls was

146 observed in only one study period and a reverse picture was observed in another post vaccination

147 period of the study. This pattern appears more likely to be due to natural variation over time rather than

148 to an increase in NVT serotypes due to community vaccination with PCV-7. 
150 All STs obtained in this study belong to the ST217 hyper virulent clonal complex responsible for

151 several epidemic outbreaks in West Africa (Leimkugel, Adams Forgor et al. 2005, Yaro, Lourd et al.

152 2006, Antonio, Hakeem et al. 2008). The prevalence of the predominant serotype 1 genotypes (ST3081

153 and ST618) varied significantly over the study period. In period 3, we were unable to detect ST618, but

154 noted instead the predominance of its quadruple locus variant ST3081. It is plausible that the changes

155 between ST618 and ST3081 in this study population provide initial evidence of an expansion of the

156 ST217 clonal complex. However, this finding could possibly have been due to temporal changes. On-

157 going invasive pneumococcal disease surveillance studies in The Gambia such as IPD surveillance in

158 the Upper River Region (Mackenzie, Plumb et al. 2012) as well as the pneumococcal disease

159 surveillance in the West Africa region consortium (personal communication, M Antonio, March 2015)

160 will provide more answers to these questions. We have also shown in our study area, the detection of a

161 new sequence type in The Gambia without evidence that this was associated with vaccination with

162 PCV-7. Such emergence of ST suggests natural variation in the molecular epidemiology of the

163 pneumococcus that requires further evaluation. A report from Brazil of a study that looked at invasive

164 serotype 1 isolates over 3 decades found temporal changes in pulse field gel electrophoresis subtypes

165 and STs over time but the effect of pneumococcal vaccination was not evaluated (Chiou, Andrade et al.

166 2008). This should be closely monitored in The Gambia in the near future as the wider PCV

167 formulation (PCV-13) has recently been introduced as part of the Expanded Programme of

168 Immunization.

169 However, we acknowledge some limitations with this study. Firstly, the samples in this study were of

170 modest size and a larger sample size would have allowed for more robust analysis between the

171 comparison groups. The modest number we got after sampling such a large population goes to support

172 the notion that serotype 1 is rare in carriage. Obtaining a much larger sample size will therefore require 
173 very large epidemiological studies and its attendant challenges. Secondly, this study was limited to

174 carriage isolates from the Western division of The Gambia. It is unclear if observations from this group

175 are applicable to a more heterogeneous population. There is therefore a need for further studies in The

176 Gambia including population-based molecular epidemiological studies assessing the distribution of

177 these STs causing IPD and whole-genome comparisons to identify genetic differences that could

178 correspond with the observed differences between otherwise highly similar strains and such studies are

179 currently underway (Williams, Loman et al. 2012).

180

181 Conclusions

182 In conclusion, we show in this study the prevalence of pneumococcal serotype 1 carriage as well as the

183 predominant genotypes and how they varied over the study periods but this did not seem related to

184 community vaccination with PCV-7. This provides important baseline data for further evaluation of 185 nasopharyngeal carriage after PCV-13 has been introduced in The Gambia.

186

187 Abbreviations

188 IPD: Invasive Pneumococcal Disease; PCV-7: 7 valent pneumococcal conjugate vaccine; NPS:

189 Nasopharyngeal Swab; MLST: Multilocus Sequence Typing; ST: Sequence type

\section{Additional Information and Declarations}

\section{Competing Interests}

193 The authors declare there are no competing interests. 


\section{Author Contributions}

196 Chinelo Ebruke conceived and designed the experiments, performed the experiments, analyzed the 197 data, wrote the paper, prepared figures and/or tables, reviewed drafts of the paper.

198 Anna Roca, Philip C Hill, Brian Greenwood, Brendan W. Wren and Richard A Adegbola conceived 199 and designed the experiments, contributed reagents/materials/analysis tools, reviewed drafts of the 200 paper.

201 Uzochukwu Egere and Ousainou Darboe contributed reagents/materials/analysis tools, reviewed drafts 202 of the paper

203 Martin Antonio conceived and designed the experiments, wrote the paper, prepared figures and/or 204 tables, reviewed drafts of the paper.

\section{Human Ethics}

206 The following information was supplied relating to ethical approvals (i.e., approving body and any 207 reference numbers):

209 Approval for this study was obtained from the Joint Medical Research Council (MRC)/Gambia

210 Government Ethics Committee and the Ethics Committee of the London School of Hygiene \& Tropical

211 Medicine, UK. The conduct of the trial was guided by a Data Safety and Monitoring Board and

212 community and individual consent was obtained from study participants.

\section{Funding}

214 Support for Chinelo Ebruke's PhD studentship and research costs was provided by the Medical

215 Research Council Unit, The Gambia. The funders had no role in study design, data collection and 216 analysis, decision to publish, or preparation of the manuscript.

\section{Acknowledgements}


219 We would like to thank all the individuals who participated in the study. We also acknowledge the use

220 of the core sequencing facility at MRC Unit, The Gambia and the S. pneumoniae MLST database

221 which is housed at Imperial College, London, UK.

222

223 


\section{References}

Adegbola, R. A., P. C. Hill, O. Secka, U. N. Ikumapayi, G. Lahai, B. M. Greenwood and T. Corrah (2006). "Serotype and antimicrobial susceptibility patterns of isolates of Streptococcus pneumoniae causing invasive disease in The Gambia 1996-2003." Trop Med Int Health 11(7): 1128-1135.

Ahern, J. W. and W. V. Raszka, Jr. (2009). "Meningitis from an uncommon serotype of Streptococcus pneumoniae in a young child." South Med J 102(11): 1189.

Antonio, M., I. Hakeem, T. Awine, O. Secka, K. Sankareh, D. Nsekpong, G. Lahai, A. Akisanya, U. Egere, G. Enwere, S. M. Zaman, P. C. Hill, T. Corrah, F. Cutts, B. M. Greenwood and R. A. Adegbola (2008). "Seasonality and outbreak of a predominant Streptococcus pneumoniae serotype 1 clone from The Gambia: expansion of ST217 hypervirulent clonal complex in West Africa." BMC Microbiol 8: 198.

Baker, C. I., C. P. Barrozo, M. A. Ryan, L. A. Pearse and K. L. Russell (2005). "Fatal meningitis in a previously healthy young adult caused by Streptococcus pneumoniae serotype 38: an emerging serotype?" BMC Infect Dis 5: 38.

Balicer, R. D., S. Zarka, H. Levine, E. Klement, T. Sela, N. Porat, N. Ash and R. Dagan (2010). "Control of Streptococcus pneumoniae serotype 5 epidemic of severe pneumonia among young army recruits by mass antibiotic treatment and vaccination." Vaccine 28(34): 5591-5596.

Bogaert, D., R. De Groot and P. W. Hermans (2004). "Streptococcus pneumoniae colonisation: the key to pneumococcal disease." Lancet Infect Dis 4(3): 144-154.

Brueggemann, A. B. and B. G. Spratt (2003). "Geographic distribution and clonal diversity of Streptococcus pneumoniae serotype 1 isolates." J Clin Microbiol 41(11): 4966-4970.

Chiou, A. C., S. S. Andrade, S. C. Almeida, R. C. Zanella, A. L. Andrade and M. C. Brandileone (2008). "Molecular assessment of invasive Streptococcus pneumoniae serotype 1 in Brazil: evidence of clonal replacement." J Med Microbiol 57(Pt 7): 839-844.

Gessner, B. D., J. E. Mueller and S. Yaro (2010). "African meningitis belt pneumococcal disease epidemiology indicates a need for an effective serotype 1 containing vaccine, including for older children and adults." BMC Infect Dis 10: 22.

Gleich, S., Y. Morad, R. Echague, J. R. Miller, J. Kornblum, J. S. Sampson and J. C. Butler (2000). "Streptococcus pneumoniae serotype 4 outbreak in a home for the aged: report and review of recent outbreaks." Infect Control Hosp Epidemiol 21(11): 711-717.

Hausdorff, W. P., J. Bryant, P. R. Paradiso and G. R. Siber (2000). "Which pneumococcal serogroups cause the most invasive disease: implications for conjugate vaccine formulation and use, part I." Clin Infect Dis 30(1): 100-121. 
Hausdorff, W. P., D. R. Feikin and K. P. Klugman (2005). "Epidemiological differences among pneumococcal serotypes." Lancet Infect Dis 5(2): 83-93.

Hill, P. C., A. Akisanya, K. Sankareh, Y. B. Cheung, M. Saaka, G. Lahai, B. M. Greenwood and R. A. Adegbola (2006). "Nasopharyngeal carriage of Streptococcus pneumoniae in Gambian villagers." $\underline{\text { Clin }}$ Infect Dis 43(6): 673-679.

Huang, S. S., R. Platt, S. L. Rifas-Shiman, S. I. Pelton, D. Goldmann and J. A. Finkelstein (2005). "Post-PCV7 changes in colonizing pneumococcal serotypes in 16 Massachusetts communities, 2001 and 2004." Pediatrics 116(3): e408-413.

Laval, C. B., A. L. de Andrade, F. C. Pimenta, J. G. de Andrade, R. M. de Oliveira, S. A. Silva, E. C. de Lima, J. L. Fabio, S. T. Casagrande and M. C. Brandileone (2006). "Serotypes of carriage and invasive isolates of Streptococcus pneumoniae in Brazilian children in the era of pneumococcal vaccines." Clin Microbiol Infect 12(1): 50-55.

Leimkugel, J., A. Adams Forgor, S. Gagneux, V. Pfluger, C. Flierl, E. Awine, M. Naegeli, J. P. Dangy, T. Smith, A. Hodgson and G. Pluschke (2005). "An outbreak of serotype 1 Streptococcus pneumoniae meningitis in northern Ghana with features that are characteristic of Neisseria meningitidis meningitis epidemics." J Infect Dis 192(2): 192-199.

Mackenzie, G. A., I. D. Plumb, S. Sambou, D. Saha, U. Uchendu, B. Akinsola, U. N. Ikumapayi, I. Baldeh, E. Usuf, K. Touray, M. Jasseh, S. R. Howie, A. Wattiaux, E. Lee, M. D. Knoll, O. S. Levine, B. M. Greenwood, R. A. Adegbola and P. C. Hill (2012). "Monitoring the introduction of pneumococcal conjugate vaccines into West Africa: design and implementation of a population-based surveillance system." PLoS Med 9(1): e1001161.

Mbelle, N., R. E. Huebner, A. D. Wasas, A. Kimura, I. Chang and K. P. Klugman (1999).

"Immunogenicity and impact on nasopharyngeal carriage of a nonavalent pneumococcal conjugate vaccine." J Infect Dis 180(4): 1171-1176.

Millar, E. V., J. P. Watt, M. A. Bronsdon, J. Dallas, R. Reid, M. Santosham and K. L. O'Brien (2008). "Indirect effect of 7-valent pneumococcal conjugate vaccine on pneumococcal colonization among unvaccinated household members." Clin Infect Dis 47(8): 989-996.

Morais, L., G. Carvalho Mda, A. Roca, B. Flannery, I. Mandomando, M. Soriano-Gabarro, B. Sigauque, P. Alonso and B. Beall (2007). "Sequential multiplex PCR for identifying pneumococcal capsular serotypes from South-Saharan African clinical isolates." J Med Microbiol 56(Pt 9): 11811184.

Nunes, S., R. Sa-Leao, L. C. Pereira and H. Lencastre (2008). "Emergence of a serotype 1 Streptococcus pneumoniae lineage colonising healthy children in Portugal in the seven-valent

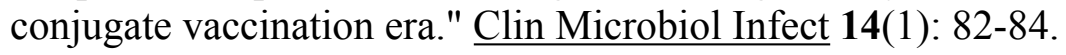

O'Brien, K. L., E. V. Millar, E. R. Zell, M. Bronsdon, R. Weatherholtz, R. Reid, J. Becenti, S. Kvamme, C. G. Whitney and M. Santosham (2007). "Effect of pneumococcal conjugate vaccine on 
314 nasopharyngeal colonization among immunized and unimmunized children in a community-

315 randomized trial." J Infect Dis 196(8): 1211-1220.

317 Roca, A., P. C. Hill, J. Townend, U. Egere, M. Antonio, A. Bojang, A. Akisanya, T. Litchfield, D. E.

318 Nsekpong, C. Oluwalana, S. R. Howie, B. Greenwood and R. A. Adegbola (2011). "Effects of

319 Community-Wide Vaccination with PCV-7 on Pneumococcal Nasopharyngeal Carriage in The

320 Gambia: A Cluster-Randomized Trial." PLoS Med 8(10): e1001107.

Smith-Vaughan, H., R. Marsh, G. Mackenzie, J. Fisher, P. S. Morris, K. Hare, G. McCallum, M. Australia." Clin Vaccine Immunol 16(2): 218-221.

Williams, T. M., N. J. Loman, C. Ebruke, D. M. Musher, R. A. Adegbola, M. J. Pallen, G. M. Weinstock and M. Antonio (2012). "Genome analysis of a highly virulent serotype 1 strain of Streptococcus pneumoniae from West Africa." PLoS One 7(10): e26742.

Yaro, S., M. Lourd, Y. Traore, B. M. Njanpop-Lafourcade, A. Sawadogo, L. Sangare, A. Hien, M. S. Ouedraogo, O. Sanou, I. Parent du Chatelet, J. L. Koeck and B. D. Gessner (2006). "Epidemiological and molecular characteristics of a highly lethal pneumococcal meningitis epidemic in Burkina Faso." Clin Infect Dis 43(6): 693-700 


\section{Figures:}

337 Figure 1: Distribution of Streptococcus pneumoniae 1 genotypes across study periods in The 338 Gambia.

339 Figure 2: Distribution of Streptococcus pneumoniae 1 genotypes across study periods in (a) 340 control and (b) vaccinated villages in The Gambia.

342 Tables

343 Table 1: Prevalence of nasopharyngeal pneumococcal serotype 1 carriage between pre-

344 vaccination study period and each of the post-vaccination study periods in The Gambia. 345

346 Table 2: Prevalence of nasopharyngeal pneumococcal serotype 1 carriage between control 347 and vaccinated villages in each study period in The Gambia. 


\section{Table $\mathbf{1}$ (on next page)}

Table 1

Prevalence of nasopharyngeal pneumococcal serotype 1 carriage between pre-vaccination study period and each of the post-vaccination study periods in The Gambia 


\begin{tabular}{|c|c|c|c|}
\hline Study period & Number of NPS & $\begin{array}{l}\text { Number of serotype } 1 \text { isolates } \\
(\%)\end{array}$ & $\begin{array}{l}P \\
\text { value }\end{array}$ \\
\hline $\begin{array}{l}\text { Pre-vaccination period } \\
\text { (December 2003- May } \\
\text { 2004) }\end{array}$ & 2746 & $13(0.47)$ & $<0.001$ \\
\hline $\begin{array}{l}\text { Post vaccination Period } 1 \\
\text { (July } 2006 \text { - March 2007) }\end{array}$ & 3986 & $9(0.23)$ & \\
\hline $\begin{array}{l}\text { Post vaccination Period } 2 \\
\text { (April } 2007 \text { - March 2008) }\end{array}$ & 3469 & $52(1.50)$ & \\
\hline $\begin{array}{l}\text { Post vaccination Period } 3 \\
\text { (April } 2008 \text { - Feb 2009) }\end{array}$ & 2118 & $13(0.61)$ & \\
\hline Total & 12,319 & $87(0.71)$ & \\
\hline
\end{tabular}




\section{Table 2 (on next page)}

Table 2

Prevalence of nasopharyngeal pneumococcal serotype 1 carriage between control and vaccinated villages in each study period in The Gambia 


\begin{tabular}{lllll}
\hline Study Period & $\begin{array}{l}\text { Village } \\
\text { Group }\end{array}$ & $\begin{array}{l}\text { Number of } \\
\text { NPS }\end{array}$ & $\begin{array}{l}\text { Number of serotype } \\
\text { 1 isolates (\%) }\end{array}$ & P value \\
\hline Pre-vaccination Period & Control & 1271 & $6(0.47)$ & 0.992 \\
& Vaccinated & 1475 & $7(0.47)$ & 0.011 \\
Post vaccination & Control & 1468 & $7(0.48)$ & \\
Period 1 & Vaccinated & 2518 & $2(0.08)$ & 0.459 \\
Post vaccination & Control & 1711 & $23(1.34)$ & \\
Period 2 & & & $29(1.65)$ & 0.004 \\
\hline Post vaccination & Control & 1171 & $2(0.17)$ & \\
Period 3 & & & $11(1.16)$ & \\
\hline
\end{tabular}


1

Figure 1

Distribution of Streptococcus pneumoniae 1 genotypes across study periods in The Gambia

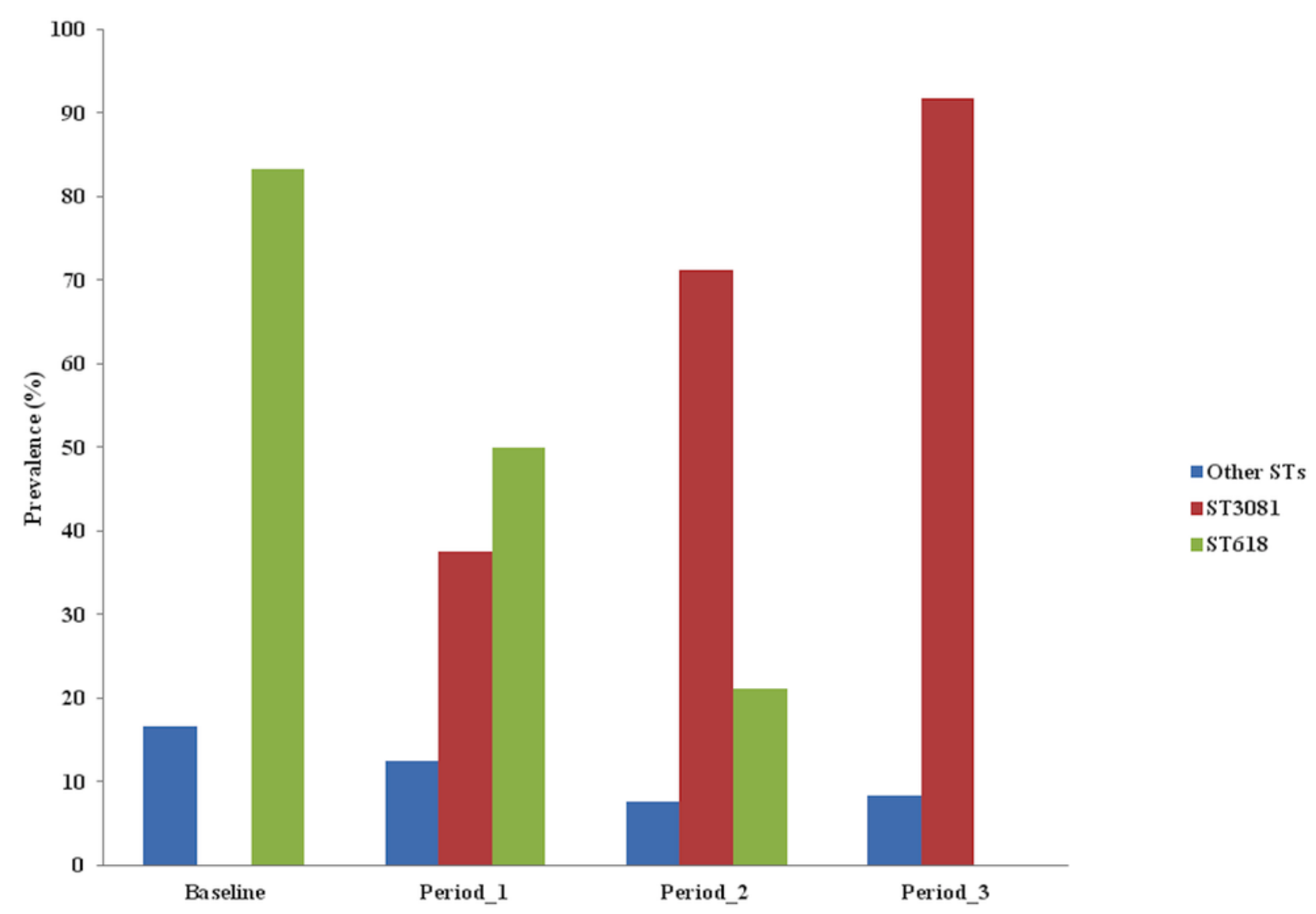

Study periods 
2

Figure 2

Distribution of Streptococcus pneumoniae 1 genotypes across study periods in (a) control and (b) vaccinated villages in The Gambia

(A)

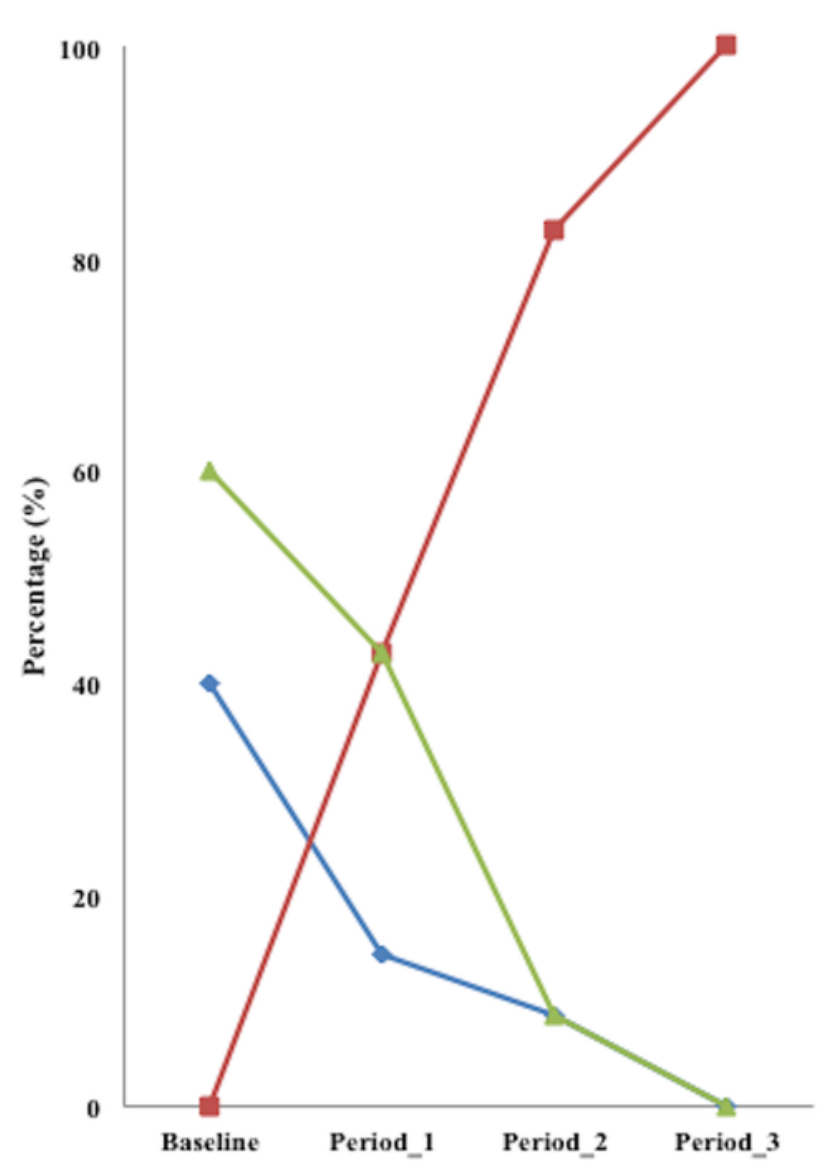

(B)

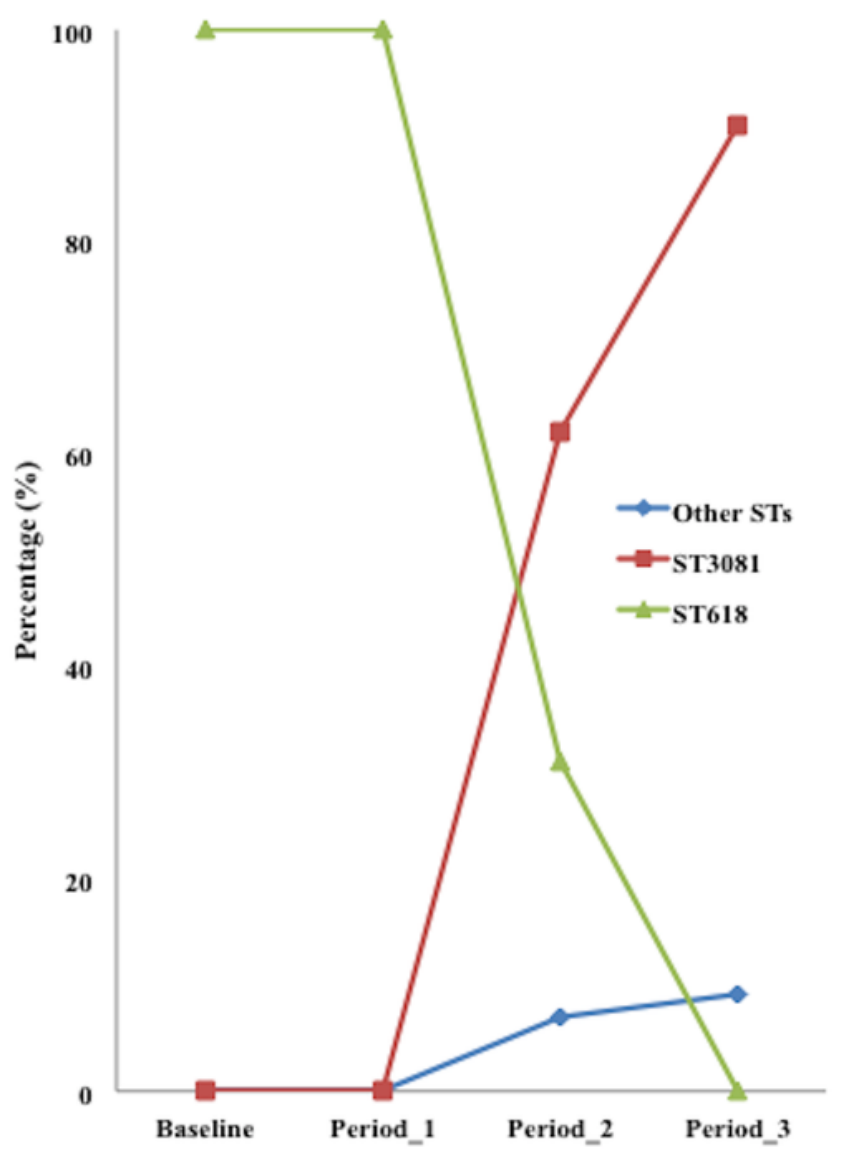

